\section{Lohnt multidisziplinäre biopsychosoziale Reha bei chronischem Rückenschmerz?}

In einem gemäß den Richtlinien der Cochrane Collaboration durchgeführten systematischen Review sollte geklärt werden, wie sich eine multidisziplinär am biopsychosozialen Modell orientierte Rehabilitation bei Patienten mit chronischem Rückenschmerz auf die Schmerzen, die allgemeine Behinderung und die Dauer der Arbeitsunfähigkeit auswirkt.

\begin{abstract}
hronischer Rückenschmerz weist eine hohe Prävalenz auf, ist für den größten Anteil der mit Behinderungen verbrachten Lebensjahre weltweit verantwortlich und mit hohen Kosten verbunden. Da monotherapeutische Ansätze in klinischen Studien nur eine eingeschränkte Wirksamkeit zeigten, wurden multidisziplinäre Interventionen, die neben physischen auch psychische, edukative und arbeitsplatzbezogene Maßnahmen enthalten, entwickelt.

Der von Kamper und Kollegen durchgeführte Review umfasste randomisierte kontrollierte Studien bei Erwachsenen mit Rückenschmerzen, die seit mindestens drei Monaten bestanden. Als multidisziplinär galten Interventionen, die zusätzlich zur physischen auch psychische und/oder arbeitsbezogene Komponenten umfassten. Kontrollinterventionen waren Standardversorgung, Physiotherapie und Operation. Als primäre Endpunkte wurden langfristige Schmerzen, Behinderungen und Arbeitsausfälle gewählt. 41 Studien mit insgesamt
\end{abstract}

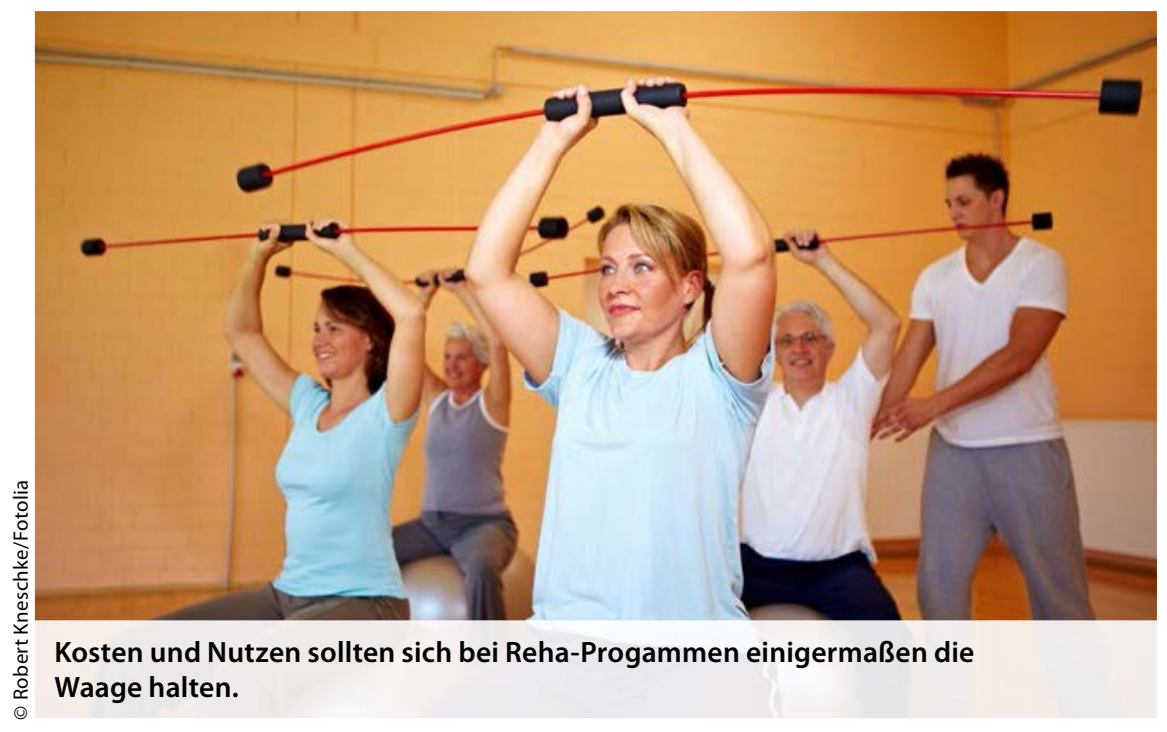

Fazit: Patienten mit chronischem Rückenschmerz profitieren in Bezug auf Schmerzen und Behinderung stärker von einer multidisziplinären Rehabilitation als von einer Standardversorgung oder Physiotherapie. Hinsichtlich Arbeitsausfällen waren MI wirksamer als Physiotherapie.

red

Kamper SJ, Apeldoorn AT, Chiarotto A et al. Multidisciplinary biopsychosocial rehabilitation for chronic low back pain: Cochrane systematic review and meta-analysis. BMJ 2015; 350: h444

6.858 Teilnehmern wurden ausgewerte $32 \%$ der Studien wiesen ein geringes Biasisiko auf, $63 \%$ berichteten vollständig über Ergebnisdaten, $39 \%$ wandten eine Intention-to-treat-Analyse an.

Multidisziplinäre Interventionen (MI) waren verglichen mit der Standardversorgung bezüglich der Schmerzen $(\mathrm{n}=821$; mäßige Studienqualität) und bezüglich der Behinderungen ( $\mathrm{n}=722$; mäßige Studienqualität) wirksamer. Bei den Arbeitsausfällen ( $\mathrm{n}=1.360$; mäßige Studienqualität) zeigte sich kein Unterschied.

Verglichen mit der Physiotherapie waren MI im Hinblick auf Schmerzen ( $\mathrm{n}=$ 872; niedrige Studienqualität) statistisch nicht signifikant wirksamer, bezüglich Behinderung ( $\mathrm{n}=1.169$; mäßige Studienqualität) und Arbeitsausfällen ( $\mathrm{n}=$ 1.006; mäßige Studienqualität) jedoch effizienter.

Gegenüber Operationen zeigten sich signifikante Unterschiede weder bei den Schmerzen noch bei den Behinderungen und den Arbeitsausfällen ( $\mathrm{n}=423$; niedrige Studienqualität).

\section{Kommentar:}

Der Cochrane Review fasst die beste derzeit zur Verfügung stehende Evidenz zur Wirksamkeit der multidisziplinären biopsychosozialen Rehabilitation bei chronischem Rückenschmerz zusammen. Multidisziplinäre Rehabilitation scheint demnach der üblichen Versorgung in Bezug auf Schmerzen und Behinderungen überlegen und im Hinblick auf Arbeitsausfälle wirksamer als alleinige Physiotherapie zu sein. Bei genauerer Betrachtung stellt sich allerdings heraus, dass die zwar statistisch signifikanten Unterschiede bezüglich einer Schmerzreduktion umgerechnet auf eine zehnstufige Skala nur einen halben Punkt betragen. Für Behinderungen entspricht der Unterschied 1,5 Punkte auf der 24-stufigen RolandMorris-Skala. Die Wahrscheinlichkeit, nach zwölf Monaten arbeitsfähig zu sein, ist für Teilnehmer an einem multidisziplinären Rehabilitationsprogramm fast doppelt so hoch wie bei alleiniger Physiotherapie.

Vergegenwärtigt man sich, dass die untersuchten biopsychosozialen Rehabilitationsprogramme kostenintensiv und mit erhöhtem Aufwand verbunden sind, so erscheint eine sorgfältige Abwägung zwischen Kosten und Nutzen solcher Interventionen dringend geboten. Da die Studienpopulation im Schnitt bereits seit mehr als einem Jahr an chronischen Schmerzen litt und mehrheitlich bereits erfolglose Therapieversuche hinter sich hatte, sollten multidisziplinäre Rehabilitationsprogramme in erster Linie Patienten mit ansonsten schlechter Prognose vorbehalten bleiben.

Dr. med. univ. Jessica T. Mattivi, MA

Klinik für Psychiatrie und Psychotherapie, Universitätsmedizin Mainz

E-Mail: jessica.mattivi@unimedizin-mainz. de 